\title{
Heat Transfer in Hydromagnetic Fluid Flow: Study of Temperature Dependence of Fluid Viscosity
}

\author{
S. K. Ghosh ${ }^{1}$, G. C. Shit $^{2 \dagger}$ and J. C. Misra ${ }^{3}$ \\ ${ }^{I}$ Department of Mathematics, Garhbeta College, Paschim Medinipore-721127, W.B., India \\ ${ }^{2}$ Department of Mathematics, Jadavpur University, Kolkata-700032, India \\ ${ }^{3}$ Institute of Technical Education \& Research, Siksha O Anusandhan University, \\ Bhubaneswar - 751030, India \\ †Corresponding Author Email: gopal_iitkgp@yahoo.co.in
}

(Received August 7, 2013; accepted December 8, 2013)

\begin{abstract}
Flow of a viscoelastic fluid through a channel with stretching walls in the presence of a magnetic field has been investigated. The viscosity of the fluid is assumed to vary with temperature. Convective heat transfer is considered along with viscous dissipation and Ohmic dissipation. The equations that govern the motion of the fluid and heat transfer are coupled and non-linear. The governing partial differential equations are reduced to a set of ordinary differential equations by using similarity transformation. The transformed equations subject to the boundary conditions are solved by developing a suitable finite difference scheme. Numerical estimates of the flow and heat transfer variables are obtained by considering blood as the working fluid. The computational values are found to be in good agreement with those of previous studies.
\end{abstract}

Keywords: MHD flow, Variable viscosity, Visco-elastic fluid, Viscous dissipation, Stretching wall, Heat transfer

\section{NOMENCLATURE}

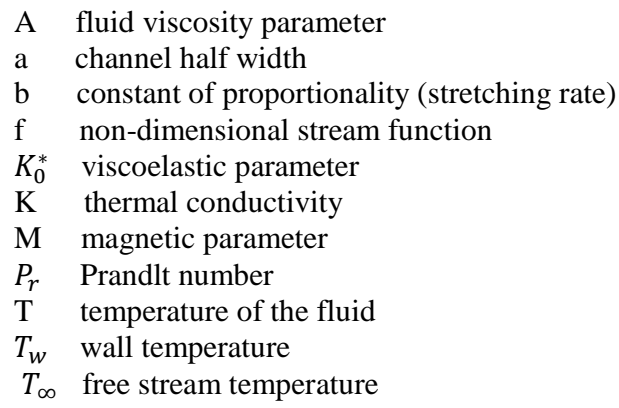

\section{INTRODUCTION}

Flow of a fluid over a moving continuous solid surface has many applications in science and engineering. Heated fluid moving between feeding rolls and wind up rolls, cooling of polymer materials by using a continuous moving sheet, glass and fiber production and manufacturing of polymeric sheets are some examples that involves flow of a viscoelastic fluid over a stretching sheet. Siddappa and Khapate (1976) performed analytical $\mathrm{u} \quad$ velocity components along $\mathrm{x}$ - direction

$\mathrm{v}$ velocity components along $\mathrm{y}$ - direction

$\eta \quad$ similarity variable

$\mu$ dynamic viscosity

$\mu^{*}$ reference viscosity

$v$ kinemetic viscosity

$\theta$ non-dimensional temperature

$\rho$ density of the fluid

$\lambda$ mixed convection parameter

denotes differentiation w.r.to $\eta$

study of the flow of Rivlin-Erickson fluid over a moving continuous solid surface. Investigation of the heat transfer during steady flow of an incompressible viscous fluid when temperature difference between the surface and the ambient fluid is proportional to some power of the distance was conducted by Carragpher et al.(1982). Rajagopal et. al (1984) presented a numerical solution for small values of the viscoelastic parameter. Introducing heat transfer, Dandapat and Gupta (1989) extended the problem considered in 
(Rajagopal et al. (1984)) and found an exact solution of the same problem. Convective flow of non-Newtonian fluids was considered by Hakiem and Abder (2006). They examined the effect of viscosity variation on flow of a micropolar fluid and found that it has substantial contribution towards change of skin friction and heat transfer. Abel and Begum (2008) carried out an analysis on MHD viscoelastic fluid flow over a stretching sheet for the case of large Prandlt number. They obtained a closed form solution by using Rosseland approximation and Kummer`s function. Alharbi et al. (2010) presented a study on viscoelastic fluid flow with heat transfer over a stretching sheet. Taking into consideration the effect of chemical reaction and thermal stratification, they made an observation that with rise in temperature, there is an increase in the values of the non-dimensional parameters like, Grashof number, Prandlt number etc. They also found that due to chemical reaction, the temperature and the velocity of the fluid reduce considerable. Assuming the viscosity and diffusivity as functions of temperature, Mukhopadhyay (2009) discussed the unsteady flow and heat transfer over a stretching sheet in the presence of wall suction. In this study the classical Runge-Kutta method was used for solving the nonlinear ordinary differential equations. A numerical study was performed by Sharidan et al. (2006) to investigate the unsteady boundary layer flow and heat transfer over a stretching sheet, while Char (1994) obtained a solution for viscoelastic fluid flow over a stretching sheet in the presence of a magnetic field with the consideration of thermal diffusion term in the energy equation. Sharma and Rao (1998), Vajravelu and Roper (1999), as well as Cortell (2006a, 2006b) investigated similar problems to explore the effects of elastic deformation and the work done thereby. In order to evaluate the influence of elastic parameter and heat transfer characteristics, Cortell (2007) studied the flow and heat transfer of a second order viscoelastic fluid by considering a non-uniform heat source, viscous dissipation and thermal radiation. $\mathrm{He}$ reported that the said parameters affect quite considerable the heat transfer and fluid flow. Elbashbeshy and Aldaqwody (2010) analyzed numerically the unsteady mixed convective flow and heat transfer over a porous stretching surface. In this study they investigated the effect of thermal radiation and magnetic field on fluid flow. Since haemoglobin contains iron oxide, blood flow is likely to be affected in the presence of a magnetic field. At the time of various diagnostics procedures, such as MRI, magnetic therapy of the arthritis patients, magnetic therapy of cancer patients, CT scan etc. human body is subject to magnetic fields of considerable high strength. Apart from this, normal human beings also sometimes have to work under the influence of electromagnetic fields when they are required to works with machines having electromagnetic component. Being motivated towards physiological fluid flows, Misra et al. (2008) and Misra and Shit (2009a) reported a study of the flow and heat transfer in a viscoelastic fluid under the action of a transverse magnetic field. They considered Walters-B fluid model to depict the fluid viscoelasticity. It was not possible to treat the problem analytically and so they developed a suitable numerical method to solve the problem. The flow of biomagnetic fluids in different situations was studied extensively by Misra and Shit (2009a, 2009b). Both these investigations were motivated towards studying the haemodynamical flow of blood in arteries. A similar problem was studied by Ray Mahapatra and Gupta (2004) that concern different types of fluid flow over a vertical plate/surface. Sharma and Singh (2009) studied the effects of variable thermal conductivity, heat source/sink on the flow of electrically conducting fluid in the presence of transverse magnetic field. They consider the heat transfer in the fluid which is passing over a stretching sheet.

The aforesaid studies are important in the sense that no investigation has been made with the consideration of viscous as well as Ohmic dissipation along with the temperature dependent viscosity. In the present paper, considering WalterB fluid model of fluid viscoelasticity, we have investigated the fluid flow in a parallel plate channel under the influence of a transverse magnetic field. In order to study the problem from more general platform the fluid viscosity has been considered as a function of temperature. It has also been assumed that velocities of plates of the channel are varying linearly with distance from the origin and that the channel has a stretching motion. To study the heat transfer in the flow region, the energy equation is considered such that it takes care of viscous and Ohmic dissipation as well as the strain energy arising out of the elastic deformation. Boundary layer approximation and similarity transformation have been used to treat the coupled non-linear partial differential equations. This set of equations is solved by the use of perturbation technique by taking the viscoelastic parameter as the perturbation parameter. The resulting non-linear ordinary differential equations are then solved by applying Newton's method with finite difference technique. The numerical results for some physical variables which are important for having an insight to understand the flow physics are presented graphically. The study bears the potential of multifold applications to different problems of engineering and industries as well as physiology 
and medicine. Hope, this model will help the medical practitioners for the diagnosis of the people suffering from fever (with high temperature) and have to undergo some scanning in the presence of high strength magnetic field such as in MRI. Moreover, any ailment persist in a physic which is surrounded by applied magnetic field may also be investigated in tune of this paper and under the context of investigated results.

\section{FORMULATION AND ANALYSIS}

Let us consider the steady laminar flow and heat transfer of an incompressible viscoelastic fluid through a channel having stretching motion, which is bounded by two parallel plates. Taking the axis of the channel as reference to $x$-axis, the upper and lower walls of the channel is designated as $y= \pm a$.

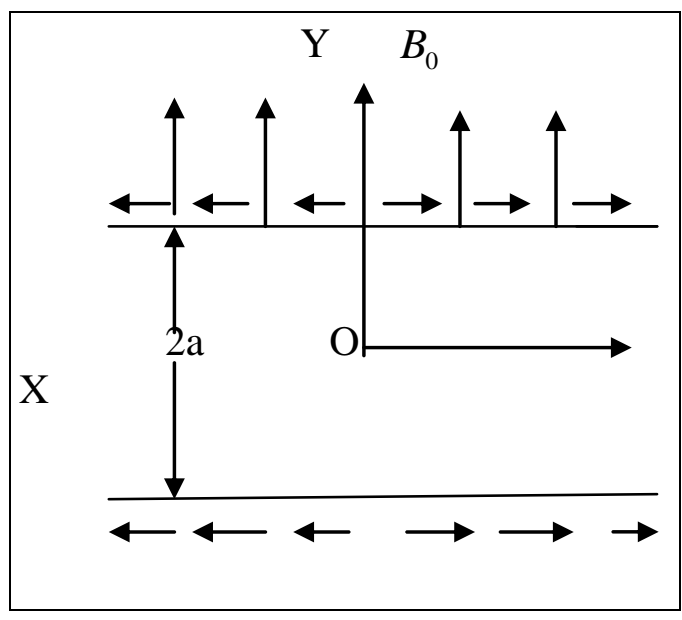

Fig.1. A sketch of the physical problem

The viscosity is considered to be temperaturedependent with Ohmic dissipation and the surface velocity of each wall to vary directly with distance from the channel axis. The motion of the fluid is driven by the stretching motion of the channel. Let us denote $B_{0}$ the strength of the applied magnetic field and $\sigma$ the electrical conductivity of the fluid. We shall consider here the steady two-dimensional motion of the fluid.

Taking into account heat transfer, the boundary layer equation with Boussinesq approximation can be presented as (cf. Misra et al (2008)),

$\frac{\partial u}{\partial x}+\frac{\partial v}{\partial y}=0$

$u \frac{\partial u}{\partial x}+v \frac{\partial u}{\partial y}=\frac{\mu}{\rho} \frac{\partial^{2} u}{\partial y^{2}}+\frac{1}{\rho} \frac{\partial u}{\partial y} \frac{\partial}{\partial y}(\mu)-\frac{\sigma B_{0}^{2} u}{\rho}$

$-\frac{k_{0}}{\rho}\left(\frac{\partial}{\partial x}\left(u \frac{\partial^{2} u}{\partial y^{2}}\right)-\frac{\partial u}{\partial y} \frac{\partial^{2} v}{\partial x \partial y}+v \frac{\partial^{3} u}{\partial y^{3}}\right)$

and
$C_{p}\left(u \cdot \frac{\partial T}{\partial x}+v \frac{\partial T}{\partial y}\right)=\frac{K}{\rho}\left(\frac{\partial^{2} T}{\partial y^{2}}\right)+\mu\left(\frac{\partial u}{\partial y}\right)^{2}$

$-\frac{k_{0}}{\rho}\left(\frac{\partial u}{\partial y}\right) \frac{\partial}{\partial y}\left(u \frac{\partial u}{\partial x}+v \frac{\partial u}{\partial y}\right)+\frac{\sigma B_{0}^{2}}{\rho} u^{2}$

where the Eq.(1) represents the mass conservation of fluid passing over any cross-section of the channel, the second term on the right hand side of (2) appears to represent the effect of variable viscosity while the second and third terms bear the effect of magnetic field force and viscoelasticity of the fluid. The second, third and fourth term on the right hand side of (3) are introduced to investigate the flow behavior due to the contribution of the viscous, viscoelastic and Ohmic dissipation respectively. Also, $u, v$ are the components of velocity of fluid along $x$ - and $y$-directions, $k_{0}$ the viscoelastic parameter, $\rho$ the density of the fluid and $\mu=\mu^{*} e^{-A T}, \mu^{*}$ being the coefficient of kinematic viscosity.

Considering the flow to be symmetric about the axis of the channel our study can be restricted to the region $0 \leq y \leq a$ only. Hence the boundary conditions may be listed as

$u=b x, v=0, T=T_{w}$ at $y=a, b>0$

and

$\frac{\partial u}{\partial y}=0, v=0, \frac{\partial T}{\partial y}=0$ at $\quad y=0$

where $b$ represents the stretching rate of the channel walls.

Let us introduce the similarity variables and nondimensional variables as follows

$u=b x f^{\prime}(\eta) ; v=-b a f(\eta)$

$\theta=\theta_{1}(\eta)+\xi^{2} \theta_{2}(\eta)$,

in which,

$\xi=\frac{x}{a}, \quad \eta=\frac{y}{a}, u^{*}=\frac{u}{a b}, \quad v^{*}=\frac{v}{a b} ;$

$\theta=\frac{T}{T_{w}}$.

Using these similarity variables in terms of the nondimensional variables in (1) - (3) we have

$$
\begin{aligned}
& f^{\prime 2}-f f^{\prime \prime}=\left(1-A_{1} \theta_{1}\right) f^{\prime \prime \prime}-M f^{\prime} \\
& -A_{1} \theta_{1}^{\prime} f^{\prime \prime}\left(1-A_{1} \theta_{1}\right)-K_{0}\left(2 f f^{\prime \prime \prime}-f f^{i v}-f^{\prime \prime 2}\right) \\
& f^{\prime \prime} \theta_{2}^{\prime}-f^{\prime \prime \prime}\left\{\theta_{2}^{\prime}-A_{1}\left(\theta_{2}^{\prime} \theta_{1}+\theta_{1}^{\prime} \theta_{2}\right)\right\}=0 \\
& \theta_{1}^{\prime \prime}+P_{r} f \theta_{1}^{\prime}=0 \\
& \theta_{2}^{\prime \prime}-P_{r}\left(2 f^{\prime} \theta_{2}-f^{\prime} \theta_{2}^{\prime}\right)-\lambda\left(1-A_{1} \theta_{1}\right) A_{1} f^{\prime \prime 2} \\
& +\lambda k_{0}\left(f f^{\prime \prime 2}-f f^{\prime \prime} f\right)+M \lambda f^{\prime 2}=0
\end{aligned}
$$

Equations (6) to (9) constitute a system of coupled non-linear ordinary differential equations. 
Considering the viscoelastic parameter $k_{0}$ to be small, we shall use the perturbation method, by taking $k_{0}$ as the perturbation parameter.

Thus we write

$f(\eta)=f_{0}(\eta)+k_{0} f_{1}(\eta)+k_{0}^{2} f_{2}(\eta)+. .$.

Using (10) in Eqs. (6) to (9), equating the coefficients of like powers of $k_{0}$ from both sides and neglecting squares and higher powers of $k_{0}$, we derive the following set of equations.

$f_{0}^{\prime 2}-f_{0} f_{0}^{\prime \prime}=\left(1-A_{1} \theta_{1}\right) f_{0}^{\prime \prime \prime}-M f \sigma^{\prime}-A_{1} \theta f_{0}^{\prime \prime}$

$2 f_{1}^{\prime} f_{0}^{\prime}-\left(f_{0} f_{1}^{\prime \prime}+f_{1} f_{0}^{\prime \prime}\right)=\left(1-A_{1} \theta_{1}\right) f_{1}^{\prime \prime \prime}-M f_{1}^{\prime}$

$-A_{1}\left(1-A_{1} \theta_{1}\right) \theta_{1}^{\prime} f_{1}^{\prime \prime}-\left(2 f_{0}^{\prime} f_{0}^{\prime \prime \prime}-f_{0} f_{0}^{i v}-f_{0}^{\prime \prime 2}\right)$

$\left(2 f_{0}^{\prime} f_{2}^{\prime}+f_{1}^{\prime 2}\right)-\left(f_{2} f_{0}^{\prime \prime}+f_{2}^{\prime \prime} f_{0}+f_{1} f_{1}^{\prime \prime}\right)$

$=f_{2}^{\prime \prime \prime}\left(1-A_{1} \theta_{1}\right)-M f_{2}^{\prime}-A_{1} \theta_{1}^{\prime}\left(1-A_{1} \theta_{1}\right) f_{2}^{\prime \prime}$

$-\left(2 f_{1}^{\prime} f_{0}^{\prime \prime}+2 f_{0}^{\prime} f_{1}^{\prime \prime \prime}-f_{0} f_{1}^{\prime \prime \prime \prime}-f_{1} f_{0}^{\prime \prime \prime \prime}-2 f_{1}^{\prime} f_{0}^{\prime}\right)$

where dimensionless parameters appearing in the Eqs. (10) to (13) are defined as

$M=\frac{\sigma B_{0}^{2}}{\rho b}, \quad \lambda=\frac{b \mu^{2}}{\rho k T_{w}}, P_{r}=\frac{\mu C_{p}}{K}$,

$K_{0}=\frac{k_{0} b}{\mu}, A=A_{1} T_{w}, \quad A_{1}=0,1,2,3$.

Moreover, the boundary conditions (4) and (5) reduce to

$f^{\prime}=1, \quad f=0, \theta=1 \quad$ at $\quad \eta=1$

and

$f^{\prime \prime}=0, f=0, \theta^{\prime}=0$ at $\eta=0$

In order to determine the flow field and the temperature field, we need to solve the Eqs.(8), (9) and (11) to (13) subject to the boundary conditions (14) and (15). It may be noted that the equations in question are highly non-linear. So we have to develop a suitable numerical procedure, describe in the section that follows.

\section{COMPUTATIONAL RESULTS AND DISCUSSION}

We develop a finite difference scheme which is stable, more accurate, efficient and relatively simple. This method consists of an iteration procedure and use of Thomas Algorithm. Thus it is very much sensitive to the initial guess. However, the problem under consideration has advantage for making an appropriate initial guess. Using Newton's linearization technique, we now proceed to solve the Eqs.(11), (12) and (13) subject to the boundary conditions (14) and (15). Using central difference scheme for the derivatives with respect to $\eta$, we can write $\left(V^{\prime}\right)_{i}=\frac{V_{i+1}-V_{i-1}}{2 \delta \eta}+0\left((\delta \eta)^{2}\right)$ and $\left(V^{\prime \prime}\right)_{i}=\frac{V_{i+1}-2 V_{i}+V_{i-1}}{(\delta \eta)^{2}}+0\left((\delta \eta)^{2}\right)$

where $V$ stands for different variables; $i$ is the gridindex in $\eta$-direction with $\eta_{i}=i * \delta \eta ; i=0,1$, $2, \ldots, m$ and $\delta \eta$ is the step size along the $\eta$-axis. Iterations are performed with the following relation $V_{i}^{n+1}=V_{i}^{n}+\left(\Delta V_{i}\right)^{n}$,

where $\left(\Delta V_{i}\right)^{n}$ represents the error at the $n$-th iteration, $i=0,1,2, m$. The stability of the numerical model is checked by reducing the step size and converges for the accuracy level of $\mathrm{O}\left(10^{-}\right.$ ${ }^{5}$ ). For the numerical computation we have used step length $\delta \eta=0.025$, further decrease in $\delta \eta$ does not bring about any significant change. A detailed description of the method can also be found in Misra et. al. (2008, 2009a, 2009b).

Here we consider an illustrative example of blood flow in arteries, by taking the values of different parameters as reported in the previous literatures. In order to get a physical insight of the problem, we intend to study the effects of various parameters like $A \in(0.0,1.5)$, a parameter related to the variable viscosity, $M \in(0,300)$ the magnetic parameter, the Prandlt number $P_{r} \in(5.0,150), K_{0}$ $\epsilon(0.0,0.2)$ the viscosity parameter and $\lambda \in(0.0$, 15) the mixed convection parameter on the viscosity, its gradient and temperature. In the first bracket, range of the parameters appear in the present investigation are articulated. The computational results are presented graphically.

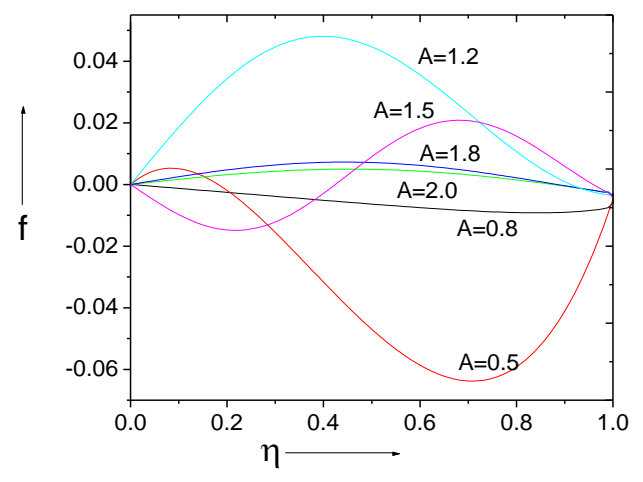

Fig. 2. The variation of stream function for

different $A$ with $\lambda=1.0, M=10.0, P_{r}=10.0$

$$
K_{0}=\mathbf{0 . 0 5}
$$

Figure. 2 gives the distribution of the fluid velocity in the channel for different values of the parameter $A$ associated with the temperature dependent viscosity. Plots corresponding to Newtonian viscosity and viscoelasticity have been shown in this figure. Taking into consideration that blood is a viscoelastic fluid one may use the results to observe 
the change in viscosity as the body temperature raised. It is seen that $0<A<1$ produce negative values of the stream function but in contrast for $A>1$ it is positive and slowly increase as the values of $A$ increases. Thus the coefficient appears in the expression of temperature dependent variable viscosity can control the flow phenomenon following a certain rule in a regular pattern. So, the parameter $A$ decelerates the motion by enhancing the frictional resistance between the fluid layers. By collecting different blood samples from different people working in hot/moderate/cold environment and meaning the viscosity of the blood samples one can have an idea of the temperature dependence of blood viscosity. The external force (Lorentz force) that creates retardation in the motion of the fluid because of its characteristics of attracting electrically conducting liquid. Hemoglobin in blood having iron gets attracted by magnetic field which results to the slow movement of fluid along the stretching sheet. Figure. 3 shows the significant effect of magnetic parameter on the function that evokes the velocity.

It is further seen that blood encounter back flow for $M>1$. The presence of a magnetic field can affect the fluid considerably. From this figure it is seen that the tendency of the back flow is enhanced as the strength of the magnetic field is increased. A comparison between the results of the present study are in good agreement with those of Misra et al. (2008).

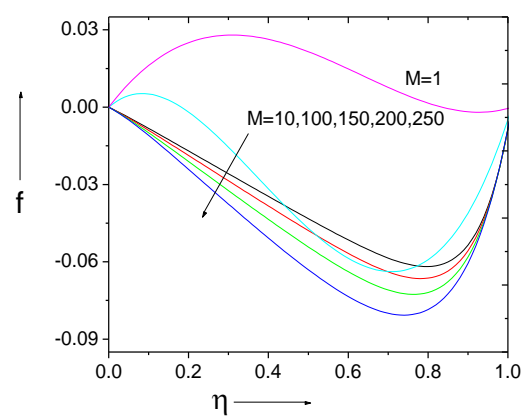

Fig. 3. The variation of stream function for different $M$ when $\lambda=1.0, A=0.5, P_{r}=\mathbf{1 0 . 0}$

$$
K_{0}=\mathbf{0 . 0 5}
$$

The graphical presentation of the results of the present study indicates that owing to the influence of the magnetic field retards the flow. From above two figures we conceive the idea that the flow may be retarded due to the some range of values of $\mathrm{A}$ and $M$. However, the values for $\mathrm{A}<0$, fluid particles displacement rate get enhanced. Hence, the parameter A has a crucial role in the movement of fluid over a stretching sheet and the motion of fluid for $\mathrm{A}<0$ is faster than $\mathrm{A}>0$ because for the values of
$\mathrm{A}<0$, viscosity decrease i.e. frictional resistance decrease.

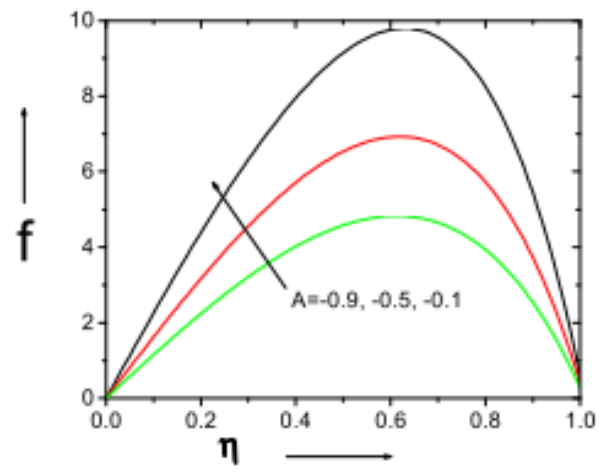

Fig. 4. Axial velocity distribution when $\mathrm{A}<0$ $\left(\lambda=1.0, M=10.0, P_{r}=10.0 K_{0}=\mathbf{0 . 0 5}\right)$

Figure. 4 gives the distribution of velocity at different locations of the channel for different values of the viscosity parameter whose value changes with the change in fluid temperature. When the fluid viscosity is low, the fluid is faster. This may be attributed to the fact that the less the viscosity of the fluid, the less is the frictional force acting on the channel walls. This causes enhancement of the flow rate.

This is a novel observation in the case of viscoelastic fluids over a stretching surface. It is interesting to note from Fig. 4 that though the fluid is viscoelastic the negative values of the parameter A significantly affects the flow behavior and the parabolic profile of Newtonian viscous flow is greatly disturbed. It may be observed further that the viscous effect suppresses the stretching effect on the flow. This suggests that the flow of industrial fluids that possess viscoelastic behavior can be changed to Newtonian flow by applying heat to the fluid in the flow regime. This mechanism can also be used to control the flow region in situations when the fluid has a tendency to coagulate leading to form a dense fluid region.

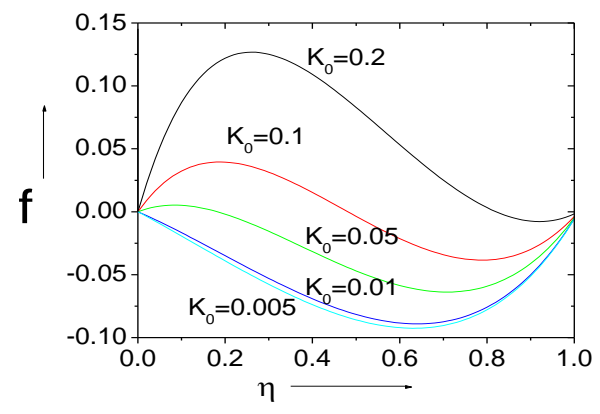

Fig. 5. Axial velocity distribution for different values of $K_{0}\left(\lambda=1.0, \mathrm{M}=10.0, P_{r}=10.0 A=0.5\right)$

Figure. 5 depicts the variation of axial velocity along with the height of the channel for different 
values of the viscoelastic parameter $K_{0}$.

Viscoelasticity is a property of the fluid which comes to the momentum equation from the constitutive equation of the fluid and to identify the effect of this property. It shows that the velocity of fluid increases as the values of the visco-elastic parameter $K_{0}$ increases. The tendency of backward flow may be attributed to the stretching motion of the surface on which the flow takes place. The appreciable change in velocity at different axial stations for each $K_{0}$ is highly sensitive as long as the non-Newtonian behavior of the fluid is dominant.

Upto this discussion we studied the appreciable change of a function which is not the axial velocity. Now Figs. 6 and 7 illustrate the variation of axial velocity f' along with the height of the channel for different values of the viscosity parameter $\mathrm{A}$ as well as the magnetic parameter M. Fig. 6 depicts the effects of viscosity parameter $A$ on the gradient of stream function. We observe that this figure has a non-rhythmic behavior in contrast to the stream function found in Fig. 2. It shows that the gradient of velocity in $y$-direction diminished with the increasing values of $A$. Moreover, for the values of $A \geq 1.8$ the velocity in $y$-direction is near about zero.

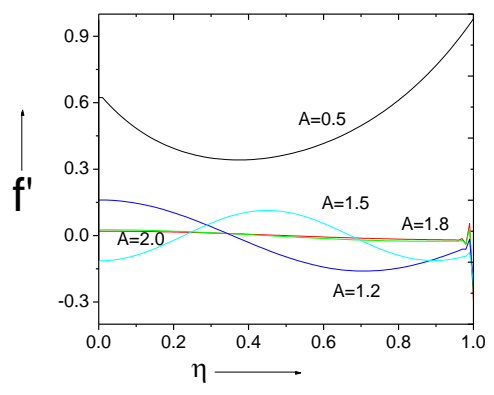

Fig. 6. Graphical representation of $f^{\prime}$ for different values of $\mathrm{A}\left(\lambda=\mathbf{1 . 0}, \mathrm{M}=\mathbf{1 0 . 0}, P_{r}=\mathbf{1 0 . 0}\right.$ $\left.K_{0}=\mathbf{0 . 0 5}\right)$

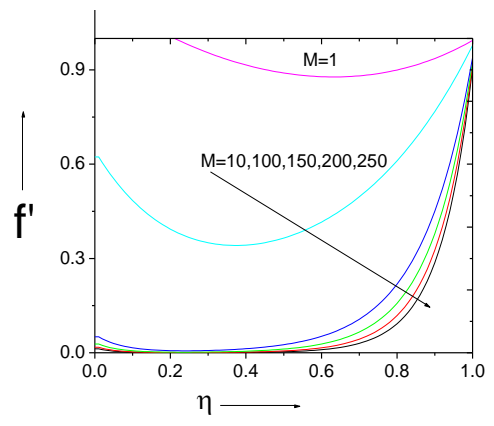

Fig.7. Graphical representation of $\boldsymbol{f}^{\prime}$ versus M $\left(\lambda=\mathbf{1 . 0}, K_{0}=\mathbf{0 . 0 5}, P_{r}=\mathbf{1 0 . 0} A=\mathbf{0 . 5}\right)$
It may be pointed out that for higher values of the viscosity parameter leads to the potential flow, where the acceleration term contribution in the computation is negligibly small. The effect of magnetic field strength on the velocity gradient in y-direction is shown in Fig. 7. From this figure we observe that as the magnetic parameter $M$ increases the axial velocity near the wall decreases gradually. The results found in this figure are good agreement with the previous studies.

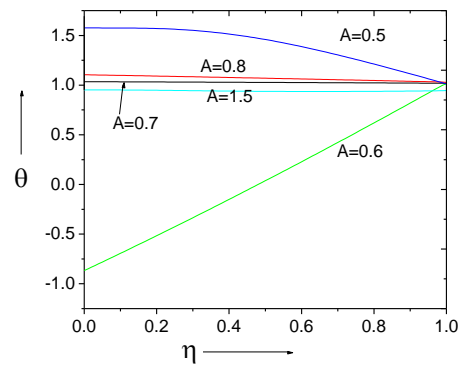

Fig. 8. Temperature distribution for different values of $A$ when $\left(\lambda=1.0, M=10.0, P_{r}=10.0\right.$

$$
\left.K_{0}=\mathbf{0 . 0 5}\right)
$$

As in the present investigation the heat transfer takes place in the fluid, we interestingly taken up the venture to study the profile due to some parameters associated with the energy equation as well as in the momentum equation. Figs. 8 to 12 represent the temperature distribution along the height of the channel for different values of the parameters involved in the present study under consideration. An arbitrary distribution of temperature is observed for various values of the viscosity parameter $A$ as shown in Fig. 8. It is interesting to note from this figure that for the higher values of $A$ temperature variation is insignificant. The heat transfer associated with the various values of $\lambda$ is depicted in Fig. 9. It shows that the parameter $\lambda$ has significant response on the temperature distribution and hence we observed that the temperature decreases with the decreasing values of $\lambda$. With a careful observation it is found that the values of $\theta$ bears same magnitude for all $\lambda \leq 1$.

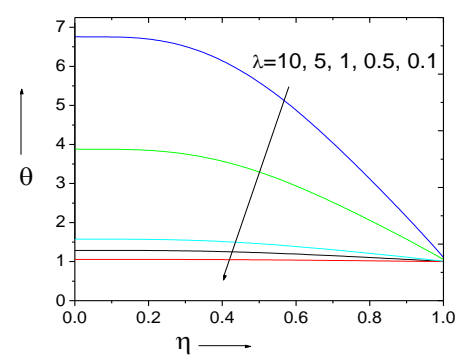

Fig. 9. Temperature distribution for different values $\lambda\left(A=\mathbf{0 . 5}, \mathrm{M}=\mathbf{1 0 . 0}, P_{r}=\mathbf{1 0 . 0} K_{0}=\mathbf{0 . 0 5}\right)$ 


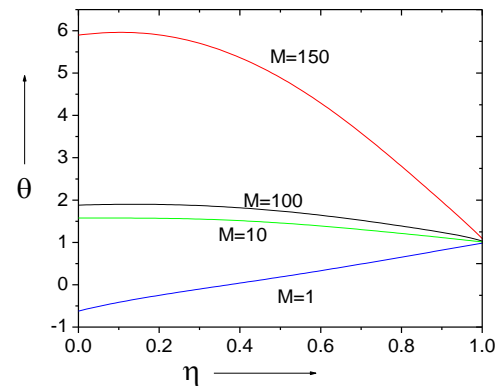

Fig. 10. Distribution of temperature $\theta$ for different values of $M\left(\lambda=\mathbf{1 . 0}, K_{0}=\mathbf{0 . 0 5}, P_{r}=\mathbf{1 0 . 0}\right.$

$$
\boldsymbol{A}=\mathbf{0 . 5})
$$

Figure 10 gives the temperature distribution along the height of the channel for different magnetic field strength in terms of magnetic parameter $M$. We observed that the temperature gradually increases with the increase of the magnetic parameter $M$. For higher values of $M$, maximum temperature occurs at the central line of the channel. It may be pointed out that the temperature difference between $M=150$ and $M=100$ is large, however between $M=100$ and $M=10$ the difference is negligible in compare to the higher values of $M$.

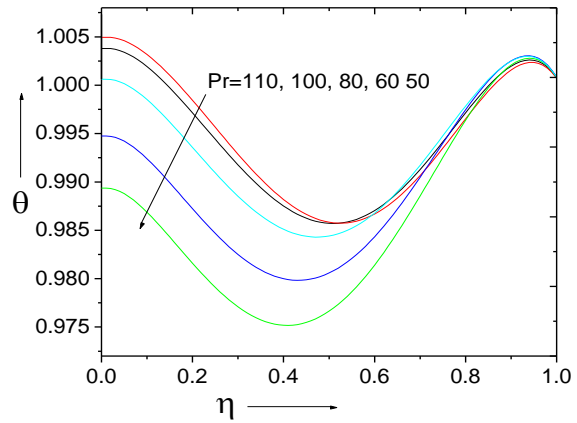

Fig. 11. Prandtl numbers effect on temperature variation $\left(\lambda=1.0, K_{0}=0.05, M=10.0 A=0.5\right)$

In Fig. 11 we present the temperature distribution for different values of the Prandatl number $\mathrm{Pr}$, an important parameter appears in the problem of fluid flow and heat transfer. We observed that the temperature decreases significantly in the central line of the channel with the increase of the Prandatl number $\mathrm{Pr}$. However, the temperature variation with Prandatl number $P r$ in the vicinity of the wall is insignificant.

The temperature distribution in the channel with the viscoelastic parameter $K_{0}$ is shown in Fig. 12. It has been shown that the temperature distribution varies in appreciable manner and moderate magnitude for different $K_{0}$. It is also found that this change in $\theta$ is significant only for small values of $K_{0}$. It may be mentioned here that the values of
$K_{0}>0.1$ absorbs heat and at different channel positions the temperature remains same. It may point out that the results found in the above figures are interesting due to its integrity in response of temperature to each values of $K_{0}$

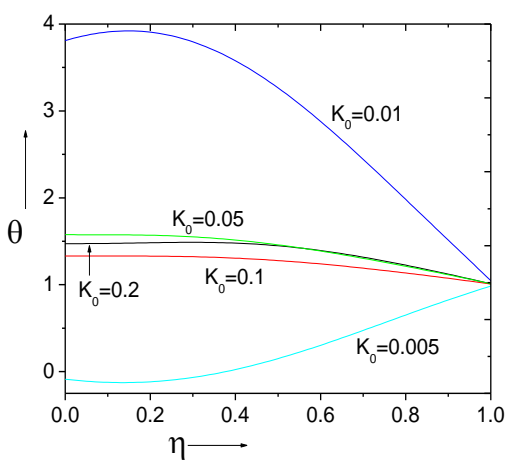

Fig. 12. Temperature profile for different values of the viscoelastic parameter $K_{0}\left(\lambda=1.0, K_{0}\right.$ $=0.05, P_{r}=10.0 M=0.5$ )

\section{CONCLUDING REMARKS}

In this paper, we have studied the effect of variable viscosity coefficient, Prandlt number, magnetic parameter and visco-elastic parameter on the fluid velocity as well as heat transfer for a visco-elastic fluid flow over a stretching sheet. The governing non-linear differential equations are solved by developing a numerical scheme, which is stable and converges for a suitable choice of initial values. There is a significant change in the velocity as well as velocity gradient for parameters like variable viscosity coefficient, magnetic parameter and viscoelastic parameter. Axial velocity of fluid increases with the decreasing values of the parameter $A$ and the magnetic parameter recede the flow with its increasing values. The similar characteristic perpetuated through the velocity gradient also. The interesting and significant results found in heat transfer are for parameters like $K_{0}, \lambda, P_{r}$ etc. The most important conclusion is that the higher values of the parameter $K_{0}$ made stagnant the heat flow in the fluid and it kept the temperature same everywhere in the channel space; however, this property is not synonymous for $\lambda$.

\section{ACKNOWLEDGEMENTS}

Authors are grateful to the esteemed reviewers for their valuable comments and suggestions for improving the paper in its current form.

The author S. K. Ghosh is thankful to the UGC (ERO), Kolkata for the financial support of this investigation under Minor Research Project (No. F. 
PSW-167/11-12). The author Professor J. C. Misra is thankful to Professor (Dr.) Manoj Ranjan Nayak, President of the Siksha O Anusandhan University, Bhubaneswar, India for his kind encouragement and for providing adequate facilities for doing research.

\section{REFERENCES}

Siddappa, B. and Khapate, B. S. (1976). RivlinErickson fluid flow past a stretching plate, Rev. Roum Sci. Tech. Mech. Appl. 21, 497-505.

Carraghper, P. and Crane, L. J. (1982). Heat transfer on a continuous stretching sheet, ZAMM, 62(10),564-565.

Rajagopal, K. R., Na, T. Y. and Gupta, A. S. (1984). Flow of viscoelastic fluid over a stretching sheet, Rheologica Acta, 23(2), 213215.

Dandapat, B. S. and Gupta, A. S (1989). Flow and heat transfer in a viscoleastic fluid over a stretching sheet, International Journal of non-Linear Mechanics, 24(3), 215--219

El-Hakiem, M. A. E and Abdou, M. M. M, (2006). The effect of variable viscosity on MHD natural convection in micropolar fluids", International Journal of Applied Mechanics in Engineering. 11(2), 301-320.

Abel, M. S. and Begum, G. (2008). Heat transfer in MHD viscoelastic fluid flow on stretching sheet with heat source/ sink, viscous dissipation, stress work and radiation for the case of large prandlt number, Chemical Engineering Communications, 195, 15031523.

Alharbi, S. M., Bazid, M.A.A. and Gendy, A. S. E. (2010). Heat and Mass transfer in MHD viscoelastic fluid flow through a porous medium over a stretching sheet with chemical reaction", Applied Mathematics, 1,446-455.

Mukhopadhyay, S. (2009). Unsteady boundary layer flow and heat transfer past a porous stretching sheet in the presence of variable viscosity and thermal diffusivity, International Journal of Heat and Mass Transfer, 52,5213$5217 .$.

Sharidan, S., Mahomood, T. and Pop, I. (2006). Similarity solution for the unsteady boundary layer flow and heat transfer due to the stretching sheet, International Journal of Applied Mechanics and Engineering., 11(3), 647-654.

Char, M. I. (1994). Heat and Mass transfer in a hydromagnetic flow of the viscoelastic fluid over a stretching sheet, Journal of Mathematical Analysis and Applications, 186,674-689.
Sharma M. S., Rao, B. N. (1998). Heat transfer in a viscoelastic fluid over a stretching sheet, Journal of Mathematical Analysis and Applications, 222, 268-275.

Vajravelu, K. Roper, T (1999). Flow and Heat transfer in a second grade fluid over a stretching sheet, International Journal of nonLinear Mechanics, 34,1031-1036.

Cortell, R (2006a). Flow and Heat transfer of a electrically conducting fluid of second grade over a stretching sheet subject to suction and to transverse magnetic field, International Journal of Heat and Mass Transfer, 49, $1851-1856$.

Cortell, R. (2006b) A note on flow and heat transfer of a viscoelastic fluid over a stretching sheet, International Journal of non-Linear Mechanics, 41,78-85.

Cortell, R. (2007) Viscoelastic fluid flow and Heat transfer over a stretching sheet under the effect of non-uniform heat source, viscous dissipation and heat radiation, International Journal of Heat and Mass Transfer, 50, 3152-3162.

Elbashbeshy, E. M. A. and Aldawody, D. A. (2010). Effects of thermal radiation and magnetic field on unsteady mixed convective flow and heat transfer over a porous stretching sheet, International Journal of Nonlinear Science, 9(4), 448-454.

Misra, J. C., Shit, G. C. and Rath, H. J. (2008). Flow and heat transfer of a MHD viscoelastic fluid in a channel with stretching walls: Some applications to haemodynamics, Computers \& Fluids, 37(1), 1-11.

Misra, J. C and Shit, G. C. (2009a) Flow of a biomagnetic viscoelastic fluid in a channel with stretching walls. Trans. ASME Journal of Applied Mechanics, 76(6), 06106:1-9.

Misra, J. C and Shit, G. C. (2009b) Biomagnetic viscoelastic fluid flow over a stretching sheet, Applied Mathematics and Computations, 210 (2), 350-361.

Ray Mahapatra, T. and Gupta, A. S. (2004). Stagnation point flow of a viscoelastic fluid towards a stretching surface, International Journal of non-Linear Mechanics, 39, 811820 .

Sharma, P. R. and Singh, G, (2009) Effects of Variable Thermal conductivity and Heat Source / Sink on MHD Flow Near a Stagnation Point on a Linearly Stretching Sheet, Journal of Applied Fluid Mechanics, vol.2,1,13-21. 